\title{
O USO DA FARINHA DE Orbignya speciosa NA FORMULAÇÃO DE ADESIVO PARA COMPENSADOS
}

\author{
THE USE OF Orbignya speciosa FLOUR IN PLYWOOD ADHESIVE MIX
}

\author{
Vanessa Coelho Almeida ${ }^{1}$ Angélica de Cássi Oliveira Carneiro ${ }^{2}$ Benedito Rocha Vital ${ }^{3}$ \\ Roberto Carlos Costa Lelis ${ }^{4}$
}

\begin{abstract}
RESUMO
O objetivo deste estudo foi avaliar o efeito da adição da farinha de babaçu nas propriedades do adesivo à base de ureia-formaldeído e compará-lo ao adesivo produzido com farinha de trigo, extensor usualmente utilizado na produção de painéis compensados. À resina ureia-formaldeído foram adicionados, em partes por massa de adesivo, 0, 10, 20 e 30\% de farinha de babaçu, e, como catalisador, sulfato de amônio, na proporção de $1,5 \%$ sobre a massa seca de sólidos da resina. Determinaram-se as seguintes propriedades dos adesivos: viscosidade, teor de sólidos, tempo de gelatinização, tempo de trabalho e pH. A farinha de babaçu apresentou propriedades semelhantes às da farinha de trigo. Ambas, de modo geral, embora tenham contribuído para o aumento da viscosidade dos adesivos, reduziram sua reatividade, pois elevaram o valor $\mathrm{pH}$, o tempo de gelatinização e o tempo de trabalho.
\end{abstract}

Palavras-chave: extensor; farinha de babaçu; ureia-formaldeído.

\begin{abstract}
The aim of this study was to evaluate the effect of the addition of babaçu flour on urea-formaldehyde adhesive properties and compare it to the adhesives produced with wheat flour, which is the extender usually used for plywood production. An amount of 0, 10, 20 and 30 parts of extender per weight of the adhesive were added. Ammonium sulfate was used as catalyst, in the proportion of $1.5 \%$ on dry weight of solid content. The following properties of the adhesive were determined: viscosity, nonvolatile content, gel time, working life and $\mathrm{pH}$. The babaçu flour presented similar properties to wheat flour. Both, in general, although contributed to the increase of the adhesives viscosity, reduced its reactivity, as increased $\mathrm{pH}$ value, gel time and working life.
\end{abstract}

Keywords: extender; filler; urea-formaldehyde.

\section{INTRODUÇÃO}

A formulação adesiva utilizada pela indústria de painéis compensados, embora seja determinada de acordo com os recursos disponíveis na região, é de extrema importância para a qualidade do produto final colado. A formulação adesiva compreende a adição de materiais como extensores, materiais de enchimento, catalizadores e água à resina, de acordo com o uso que será dado ao painel compensado produzido. E para avaliar a qualidade do adesivo formulado, antes de sua utilização propriamente dita, a avaliação de propriedades como viscosidade, teor de sólidos e $\mathrm{pH}$, dentre outras, é indispensável para prever o seu comportamento durante o processo de colagem.

1 Engenheira Florestal, Doutoranda pelo Curso de Pós-Graduação em Engenharia Florestal, Universidade Federal do Paraná, Av. Prefeito Lothário Meissner, 632, Jardim Botânico, CEP 80210-170, Curitiba (PR), Brasil. vanessaufrrj@yahoo.com.br.

2 Engenheira Florestal, Dra., Professora do Departamento de Engenharia Florestal, Universidade Federal de Viçosa, CEP 36570-000, Viçosa (MG), Brasil. cassiacarneiro@ufv.br.

3 Engenheiro Florestal, Dr., Professor do Departamento de Engenharia Florestal, Universidade Federal de Viçosa, CEP 36570 000, Viçosa (MG), Brasil. bvital@ufv.br.

4 Engenheiro Florestal, Dr., Professor do Departamento de Produtos Florestais, Instituto de Florestas, Universidade Federal Rural do Rio de Janeiro, CEP 23890-000, Seropédica (RJ), Brasil. r.lelis@ufrrj.br

Recebido para publicação em 15/05/2010 e aceito em 22/11/2012

Ci. Fl., v. 24, n. 1, jan.-mar., 2014 
Há muitos anos, a farinha de trigo é o extensor mais utilizado pelas indústrias de painéis compensados, cujo objetivo principal é a redução dos custos de produção. Além da importância econômica, a adição de farinha de trigo proporciona viscosidade adequada tanto para a aplicação dos adesivos, quanto durante a fase inicial de aquecimento do painel na prensa.

Embora seja empregada em larga escala e seu preço seja apreciável na maioria das regiões do Brasil, a farinha de trigo tem grande importância na alimentação humana e de animais, onde é empregada há milhares de anos. É constante a busca por extensores que possam substituir a farinha de trigo no processo de produção de compensados, como observado nas pesquisas realizadas por Moreira (1985), Iwakiri et al. (2000) e Cardoso \& Aguiar (2003).

Segundo a Companhia Nacional de Abastecimento (CONAB), a produção nacional estimada para safra 2009/2010 está em 5.095.300,0 toneladas de trigo. Embora elevada, a produção nacional está distribuída em apenas oito dos vinte e sete estados da Federação e o preço da saca do trigo de $60 \mathrm{~kg}$ varia conforme a região (CONAB, 2009).

Segundo TOBASA (2009), na Região Sul, há empresas que fazem uso da farinha de babaçu como extensor, substituindo em até $55 \%$ a farinha de trigo no processo de produção de painéis compensados.

A farinha de babaçu é um resíduo da utilização do fruto da palmeira Orbignya speciosa (Mart.), nativa da Região Norte do Brasil. O óleo de coco extraído da amêndoa é utilizado na indústria cosmética, alimentícia, para produção de sabão de coco, detergentes e lubrificantes (CAMPESTRE, 2008) e o resíduo (farinha de babaçu) tem sido introduzido no mercado nacional como extensor para utilização na indústria de painéis de madeira compensada (FERREIRA et al., 2009; RIBEIRO, 2008).

Diante da escassez de informação técnica sobre o uso da farinha de babaçu na formulação de adesivos para a indústria de compensados, esta pesquisa teve como objetivo avaliar o efeito da adição da farinha de babaçu nas propriedades do adesivo à base de ureia-formaldeído.

\section{MATERIAL E MÉTODOS}

\section{Materiais}

Utilizou-se a resina ureia-formaldeído
Cascamite ${ }^{\circledR}$ 5-H, produzida pela Borden Chemical, atual Momentive Química do Brasil Ltda., com as seguintes especificações técnicas: viscosidade Brookfield de 400 a $1000 \mathrm{cP}, \mathrm{pH}$ de 7,4 a 7,8, densidade de 1,28 a $1,30 \mathrm{~g} / \mathrm{cm}^{3}$ e teor de sólidos de 64 a 66\% (BORDEN QUÍMICA, 2007). Como catalisador, empregou-se o sulfato de amônio $\left(\mathrm{NH}_{4}\right)_{2} \mathrm{SO}_{4}$, em solução a $20 \%$, na proporção de $1,5 \%$ sobre o teor de sólidos do adesivo.

Como extensores, utilizaram-se a farinha de trigo, de granulometria $98,7 \%$, passante em peneira de 250 mesh, produzida pela empresa Moinhos Vera Cruz e a farinha de babaçu, fornecida pela empresa TOBASA Bioindustrial de Babaçu S.A., da cidade de Tocantinópolis - TO, que apresentou granulometria $96,4 \%$, passante em peneira de 250 mesh (Figura 1).

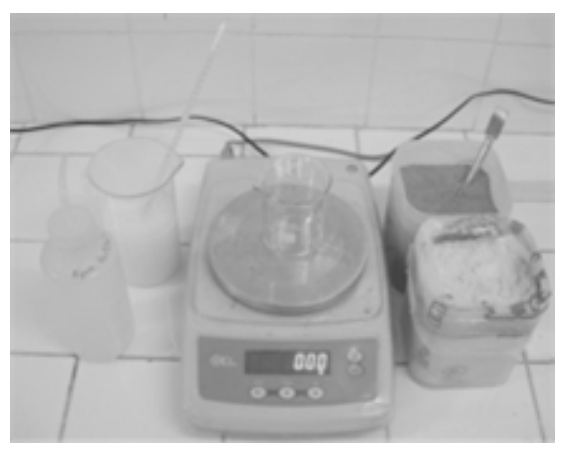

FIGURA 1: Materiais utilizados na composição adesiva.

FIGURE 1: Materials used in the adhesive compo sition.

As farinhas de trigo e de babaçu foram empregadas na formulação de diferentes misturas com o adesivo ureia-formaldeído, totalizando sete tratamentos, apresentados na Tabela 1.

As farinhas foram diluídas em água para compor os tratamentos. A quantidade de água

TABELA 1: Plano experimental.

TABLE 1: Experimental plan.

\begin{tabular}{cc}
\hline Tratamento & Extensor \\
\hline 1 & $0 \%$ \\
2 & $10 \%$ Farinha de trigo \\
3 & $20 \%$ Farinha de trigo \\
4 & $30 \%$ Farinha de trigo \\
5 & $10 \%$ Farinha de babaçu \\
6 & $20 \%$ Farinha de babaçu \\
7 & $30 \%$ Farinha de babaçu \\
\hline
\end{tabular}


utilizada correspondeu a $50 \%$ da quantidade de extensor adicionado ao adesivo. Este procedimento foi adotado após testes preliminares, quando se observou que o excesso de água, frequentemente, provocava delaminações nos painéis.

\section{Metodologia}

Para avaliar o efeito da adição de farinha de babaçu e de trigo no adesivo foram determinadas as seguintes propriedades: teor de sólidos, viscosidade, tempo de gelatinização, tempo de trabalho e $\mathrm{pH}$. Para cada propriedade foram feitas quatro repetições.

Para a determinação do teor de sólidos, que pode ser entendido como a parte do adesivo que forma a linha de cola propriamente dita, amostras de aproximadamente $2 \mathrm{~g}$ de adesivo foram levadas à estufa com ventilação natural a $105 \pm 1^{\circ} \mathrm{C}$ até atingir peso constante $( \pm 3 \mathrm{~h})$. Pela razão entre a massa da amostra de adesivo e o resíduo sólido resultante, calculou-se o teor de sólidos conforme a norma ASTM 1490-01 (2006).

A determinação da viscosidade dos adesivos, baseada na norma americana ASTM D 1084-97 (método B), foi feita num viscosímetro Brookfield, que consiste em um agitador rotativo que mede a viscosidade do fluido com base na resistência à agitação. O "spindle" (agitador) empregado para a determinação da viscosidade dos adesivos foi de número 3 , com velocidade de rotação de $12 \mathrm{rpm}$. Para todos os tratamentos, a viscosidade foi determinada após 90 segundos do início da rotação do "spindle".

O tempo de gelatinização foi obtido de acordo com Carneiro (2002). Amostras de $1 \mathrm{~g}$ de adesivo foram depositadas em tubos de ensaio aquecidos a $150^{\circ} \mathrm{C}$ no aparelho Gel-timer ${ }^{\circledR}$. Cronometrou-se o tempo gasto para a gelatinização do adesivo, que corresponde ao período transcorrido desde a preparação do adesivo até a fase de gel que caracteriza a transição do estado líquido para o estado sólido. A temperatura de $150^{\circ} \mathrm{C}$ para a determinação do tempo de gelatinização foi escolhida após estudos preliminares, através da técnica termoanalítica Calorimetria Exploratória Diferencial (DSC).

O tempo de trabalho, que se refere ao tempo de vida útil do adesivo preparado, foi determinado com o auxílio de um cronômetro, acionado após a adição do catalisador em cada amostra de 15 $\mathrm{mL}$ de adesivo mantido a temperatura de $25^{\circ} \mathrm{C}$. A contagem do tempo de trabalho foi cessada quando o adesivo deixou de ser utilizável, devido à elevada viscosidade e perda da pegajosidade.

$\mathrm{O}$ valor $\mathrm{pH}$ dos adesivos foi medido a $25^{\circ} \mathrm{C}$ em um pH-metro calibrado. Esta temperatura foi mantida para a determinação das demais propriedades dos adesivos.

\section{Análise estatística}

Para a análise das propriedades dos adesivos, o experimento foi instalado segundo um delineamento inteiramente casualizado (DIC) com quatro repetições. Os dados foram submetidos à análise de variância. E os efeitos da adição de farinha de babaçu e de trigo, quando significativos a $5 \%$, tiveram suas médias ajustadas por regressão.

\section{RESULTADOS E DISCUSSÃO}

\section{Teor de sólidos}

A análise de variância indicou que as adições de farinha de babaçu tiveram efeito significativo no teor de sólidos dos adesivos, pelo teste $\mathrm{F}$ a $5 \%$ de probabilidade. Entretanto, as adições de farinha de trigo não foram significativas, apresentando valor médio de teor de sólidos de 58,37\%.

A variação do teor de sólidos, em função das porcentagens de farinha de babaçu, é apresentada na Figura 2, onde se observa o efeito estimado e observado, bem como a equação obtida por análise de regressão, com o respectivo coeficiente de determinação.

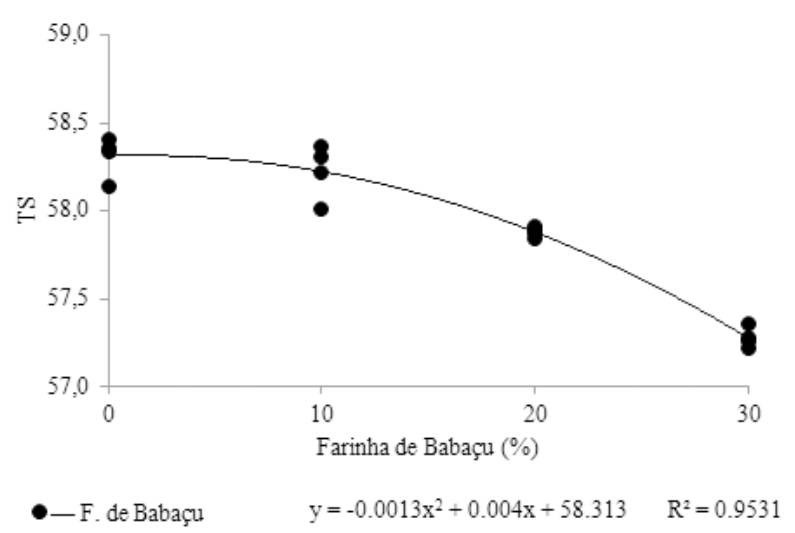

FIGURA 2: Variação do teor de sólidos (TS) dos adesivos, em função da adição de farinha de babaçu.

FIGURE 2: Adhesive nonvolatile content variation, according to the addition of babaçu flour. 
O teor de sólidos, que corresponde à porcentagem, em massa, do material não volátil contido no adesivo, decresceu com a adição de farinha de babaçu devido à diluição em água.

Embora estatisticamente haja diferença significativa entre os valores médios encontrados para o teor de sólidos dos adesivos após a adição de farinha de babaçu, a variação foi apenas de 58,37 a 57,01\%, o que, em termos práticos, não tem influência expressiva na qualidade da linha de cola.

\section{Viscosidade}

A análise de variância indicou que as adições dos extensores (farinha de trigo e farinha de babaçu) tiveram efeito na viscosidade dos adesivos, pelo teste $\mathrm{F}$ a $5 \%$ de probabilidade. A variação da viscosidade, em função da adição de farinha de babaçu e de trigo, é apresentada na Figura 3.

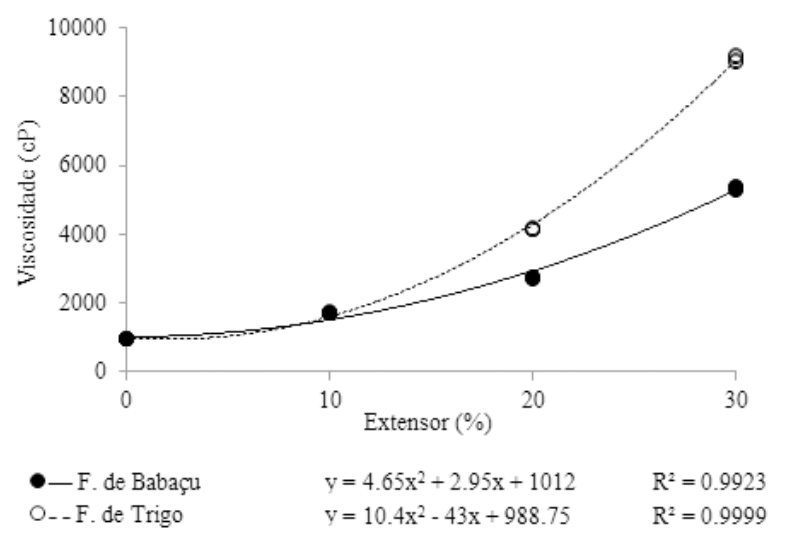

FIGURA 3: Variação da viscosidade dos adesivos em função da adição de farinha de babaçu e de trigo.

FIGURE 3: Adhesive viscosity variation according to addition of babaçu and wheat flour.

Conforme pode ser observado na Figura 3, as farinhas de trigo e de babaçu contribuíram de modo significativo para o aumento da viscosidade. Entretanto, durante o desenvolvimento deste trabalho, observou-se que não houve dificuldade para aplicação dos adesivos com espátula para estes tratamentos estudados, compostos por até $30 \%$ de extensor.

Segundo Iwakiri (2005), os extensores devem manter a viscosidade do adesivo entre 4000 e $6500 \mathrm{cP}$, para que ele seja utilizado na produção de painéis compensados. Considerando este parâmetro, apenas as formulações com $20 \%$ de farinha de trigo e as com $30 \%$ de farinha de babaçu, seriam aplicáveis na indústria de painéis compensados.

A viabilidade de utilização dos adesivos dependerá do modo de aplicação. A maioria das empresas que produz painel compensado utiliza o aplicador de rolos para a transferência do adesivo para a lâmina. Este equipamento possibilita a aplicação de adesivos com viscosidades variadas, sendo a gramatura controlada pela distância entre os rolos e a lâmina.

O acréscimo de farinha de trigo resultou em maior viscosidade relativa quando comparado ao acréscimo de farinha de babaçu. Provavelmente porque a farinha de trigo possui maior teor de amido (65 a 70\%), quando comparada à farinha de babaçu (55 a $62 \%)$.

\section{Tempo de gelatinização}

A análise de variância indicou que as adições de farinha de babaçu e de trigo tiveram efeito no tempo de gelatinização dos adesivos, pelo teste F a 5\% de probabilidade. As variações do tempo de gelatinização, em função das porcentagens de farinha de babaçu e de trigo adicionadas ao adesivo, estão apresentadas na Figura 4.

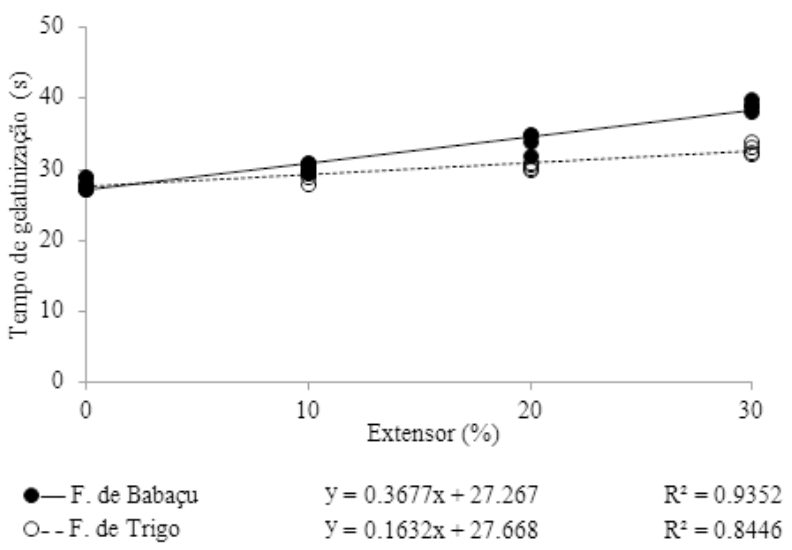

FIGURA 4: Variação de tempo de gelatinização dos adesivos em função da adição de farinha de babaçu e de trigo.

FIGURE 4: Adhesive gel time variation according to addition of babaçu and wheat flour.

Observa-se na Figura 4 que, quanto maior a quantidade de extensor, maior foi o tempo de gelatinização, portanto, menor a reatividade dos adesivos. O tempo de gelatinização aumentou 11 segundos após a adição de $30 \%$ de farinha de babaçu e apenas 5 segundos, após a adição de farinha de trigo, indicando a maior interação entre a farinha trigo e a resina ureica, devido ao seu maior teor de 
amido.

Tempo de gelatinização muito reduzido, associado à alta viscosidade dos adesivos, não é uma condição adequada para a indústria de compensados, visto que pode ocorrer a pré-cura do adesivo antes que aconteçam as funções de movimento e mobilidade, acarretando a formação de linha de cola espessa.

Para os adesivos de baixa viscosidade, o movimento de penetração na estrutura porosa da madeira é mais rápido. Neste caso, a rápida gelatinização pode ser favorável para evitar a excessiva penetração do adesivo na madeira, além de reduzir o tempo de prensagem.

\section{Tempo de trabalho}

A análise de variância indicou que as adições dos extensores tiveram efeito no tempo de trabalho dos adesivos, pelo teste $\mathrm{F}$ a $5 \%$ de probabilidade. Conforme pode ser observado na Figura 5, a vida útil ou o tempo de utilização do adesivo aumentou com a adição dos extensores.

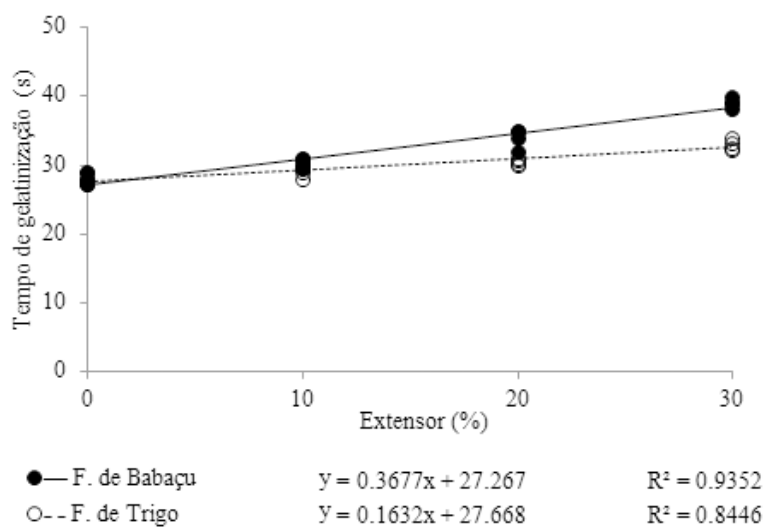

FIGURA 5: Variação de tempo de trabalho dos adesivos em função da adição de farinha de babaçu e de trigo.

FIGURE 5: Variation of adhesive working life, according to addition of babaçu and wheat flour.

O tempo de trabalho foi sutilmente menor para os adesivos formulados com farinha de trigo, principalmente para os adesivos com pequenas proporções de extensor. Este comportamento condiz com a maior reatividade da farinha de trigo, embora esta diferença entre os dois extensores não necessite ser considerada na prática de produção de painéis compensados.

De modo geral, o aumento do tempo de trabalho é favorável ao processo de fabricação de painéis compensados, pois a rápida polimerização do adesivo à temperatura ambiente poderia ocasionar perda do painel antes deste ser levado à prensa.

Neste experimento, o adesivo formulado sem extensor (tratamento 1) apresentou tempo de trabalho de 31,13 minutos. Os maiores valores de tempo de trabalho foram de 94,76 e 95,01 minutos para os adesivos formulados com $30 \%$ de farinha de trigo e de babaçu, respectivamente.

\section{pH}

$\mathrm{O}$ adesivo de ureia-formaldeído puro (sem catalisador) apresentou pH 6,85. Com a adição do sulfato de amônia (tratamento 1) o $\mathrm{pH}$ foi reduzido para 4,64.

A análise de variância indicou que as adições de farinha de babaçu e de trigo tiveram efeito no $\mathrm{pH}$ dos adesivos, pelo teste $\mathrm{F}$ a $5 \%$ de probabilidade. Através da Figura 6, pode-se observar que, aumentando as quantidades dos extensores na formulação do adesivo, ocorre aumento do $\mathrm{pH}$.

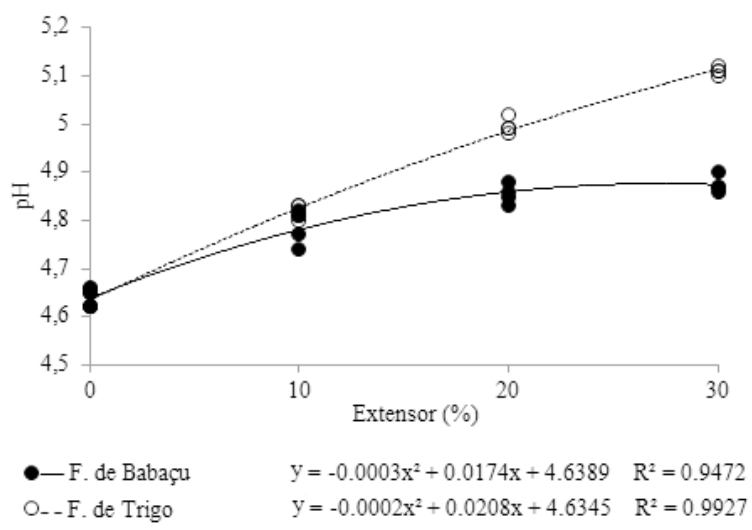

FIGURA 6: Variação de $\mathrm{pH}$ dos adesivos, em função da adição de farinha de babaçu e de trigo.

FIGURE 6: Variation of adhesive $\mathrm{pH}$, according to the addition of babaçu and wheat flour.

$\mathrm{O}$ aumento no $\mathrm{pH}$ dos adesivos foi maior quando se adicionou a farinha de trigo, evidenciando seu caráter mais básico, tendo, os produzidos com $30 \%$ de extensor, maior $\mathrm{pH}$.

Para os adesivos à base de ureia-formaldeído, quanto mais ácida a solução do adesivo, mais rápida será a cura (MOREIRA, 1985). Isso é evidenciado neste trabalho quando se compara a variação do tempo de gelatinização e do tempo de trabalho e 
a variação do $\mathrm{pH}$. De modo geral, quanto menor a quantidade de extensor adicionado, menor foi o $\mathrm{pH}$, o tempo de trabalho e o tempo de gelatinização, ou seja, maior a reatividade do adesivo, tanto a $150^{\circ} \mathrm{C}$ quanto à temperatura ambiente.

\section{CONCLUSÕES}

Os adesivos formulados com farinha de babaçu apresentaram propriedades semelhantes aos formulados com farinha de trigo. Ambos os extensores contribuíram para o aumento da viscosidade, valor $\mathrm{pH}$, tempo de gelatinização e do tempo de trabalho dos adesivos à base de ureiaformaldeído. Entretanto, reduziram sua reatividade e o teor de sólidos.

Considerando o aumento da viscosidade causado pela adição dos extensores, as formulações com até $20 \%$ de farinha de trigo e $30 \%$ de farinha de babaçu, podem ser utilizadas na indústria de painéis compensados.

Embora a redução da reatividade dos adesivos e o aumento da viscosidade sejam considerados efeitos negativos, comuns após a adição de extensores, em conjunto, podem tornarse um efeito positivo para evitar a cura do adesivo na superfície da madeira antes de sua penetração, ou seja, essa combinação de menor reatividade e maior viscosidade pode evitar a formação de linhas de cola espessas.

A redução do teor de sólidos provocada pela adição de farinha de babaçu, não é significativa em termos práticos. $\mathrm{O}$ aumento do $\mathrm{pH}$ em 0.28 porcentuais também não deve ter efeito significativo em processos industriais.

Considerando os parâmetros de colagem aqui estudados, pode-se concluir que a farinha de babaçu (na proporção de até $30 \%$ ) pode ser indicada como extensor substituto da farinha de trigo em indústrias de painéis compensados.

\section{AGRADECIMENTOS}

À Coordenação de Aperfeiçoamento de Pessoal de Nível Superior (CAPES), pelo auxílio financeiro e por incentivar a pesquisa científica no Brasil.

\section{REFERÊNCIAS BIBLIOGRÁFICAS}

AMERICAN STANDARD OF TESTING METHODS. ASTM D 1084 - 97. Standard Test Methods for Viscosity of Adhesives. West Conshohocken,
PA, 1997.

AMERICAN STANDARD OF TESTING METHODS. ASTM D 1490-01 (2006). Standard Test Method for Nonvolatile Content of Urea-Formaldehyde Resin Solutions. West Conshohocken, PA, 2006. BORDEN QUÍMICA E COMÉRCIO LTDA Boletim técnico para adesivos cascamite 5H. Prensa quente. Curitiba, 2007. 5 p. CAMPESTRE INDÚSTRIA DE ÓLEOS VEGETAIS LTDA. Babaçu. Disponível em: $<$ (http:// www.campestre.com.br/oleo-de-coco-babacu.sht$\mathrm{ml})>$ Acesso em: 15 de setembro de 2008.

CARDOSO, E. M. R.; AGUIAR, O. J. R. de. Utilização da mandioca na indústria de compensados de madeira. Belém: Embrapa Amazônia Oriental, 2003. 4 p. (Embrapa Amazônia Oriental. Comunicado técnico, 80).

CARNEIRO, A. C. O. Efeito da sulfitação dos taninos de E. grandis e E. pellita para produção de chapas de flocos. 2002. 90 f. Dissertação (Mestrado em Ciência Florestal)-Universidade Federal de Viçosa. Viçosa, 2002.

CONAB - Companhia Nacional de Abastecimento. Acompanhamento de safra brasileira: grãos, terceiro levantamento, dezembro 2009. Companhia Nacional de Abastecimento, Brasília: Conab, 2009. Disponível em: <(http://www.conab.gov. $\mathrm{br} /$ conabweb/download/safra/3graos 09.12.pdf)> Acesso em: 01 de janeiro de 2010.

FERREIRA, E. da. S. et al. Utilização da farinha de babaçu como extensor alternativo na produção de painéis compensados. Ciência Florestal, Santa Maria, v. 19, n. 3, p. 329-333, jul./set. 2009.

IWAKIRI, S. Painéis de madeira reconstituída. Curitiba: FUPEF, 2005. 247 p.

IWAKIRI, S. et al. Utilização de extensores alternativos na produção de compensados multilaminados. Ciência Florestal, Santa Maria, v. 10, n. 1, p. 77-83, 2000.

MOREIRA, W. S. Extensores alternativos para produção de compensados com resina ureiaformaldeído. 1985. 81 f. Dissertação (Mestrado em Ciência Florestal) - Universidade Federal do Paraná, Curitiba, 1985.

RIBEIRO, T. S. Produção de painéis compensados de Pinus taeda com resina ureia-formaldeído utilizando diferentes extensores. 2008. $18 \mathrm{f}$. Monografia (Graduação em Engenharia Florestal) - Universidade Federal Rural do Rio de Janeiro, Seropédica, 2008.

TOBASA - Tobasa Bioindustrial de Babaçu S.A. Comunicação verbal. Jan./dez. 2009. 